\title{
Time-Temperature Superposition of Structural Relaxation in a Viscous Metallic Liquid
}

\author{
A. Meyer* \\ National Institute of Standards and Technology, NIST Center for Neutron Research, Gaithersburg, Maryland 20899 \\ and University of Maryland, Department for Materials and Nuclear Engineering, College Park, Maryland 20742 \\ R. Busch ${ }^{\dagger}$ \\ California Institute of Technology, W. M. Keck Laboratories of Engineering Materials, 138-78, Pasadena, California 91125 \\ H. Schober \\ Institut Laue-Langevin, 38042 Grenoble, France
}

(Received 12 March 1999)

\begin{abstract}
Bulk metallic glass-forming $\mathrm{Pd}_{40} \mathrm{Ni}_{10} \mathrm{Cu}_{30} \mathrm{P}_{20}$ has been investigated in its equilibrium liquid by quasielastic neutron scattering. The quasielastic signal exhibits a structural relaxation as known from nonmetallic viscous liquids. Even well above the melting point, the structural relaxation is nonexponential and obeys a universal time-temperature superposition. From the mean relaxation times average diffusivities have been determined, resulting in values on a $10^{-10} \mathrm{~m}^{2} \mathrm{~s}^{-1}$ scale, 3 orders of magnitude slower than in simple metallic liquids.

PACS numbers: 61.25.Mv, 61.12.-q, 61.20.Lc, 64.70.Pf
\end{abstract}

During the past few years, new multicomponent $\mathrm{Zr}$ based alloys have been found which show a high stability against crystallization in their undercooled liquid state when cooled below their melting point as well as when heated above the calorimetric glass transition temperature [1,2]. Cooling rates as low as $1 \mathrm{~K} / \mathrm{s}$ allow for the casting of bulk metallic glass of up to $50 \mathrm{~mm}$ in the smallest dimension. The stability with respect to crystallization comes along with a viscosity of several $\mathrm{Pa} \mathrm{s}$ at their liquidus temperatures [3,4], which is some 3 orders of magnitude larger than in simple metallic liquids. Hence, one expects atomic motion in the liquid state to differ from the microscopic dynamics in simple metals [5].

Recently, we investigated the fast dynamics in liquid $\mathrm{Zr}_{46.8} \mathrm{Ti}_{8.2} \mathrm{Cu}_{7.5} \mathrm{Ni}_{10} \mathrm{Be}_{27.5}$ (V4) by inelastic neutron scattering [6]. Our results compare well to the behavior of certain molecular and ionic glass-forming liquids [7], and to predictions by the mode-coupling theory (MCT) of the liquid-to-glass transition [8]. Within mode-coupling theory, a fast relaxation process on a $\mathrm{meV}$ scale-the fast $\beta$ relaxation, which can be visualized as a rattling of the atoms in the transient cages formed by their neighbors prepares structural $\alpha$ relaxation responsible for viscous flow. It turned out, that, indeed, there is a fast $\beta$ relaxation in liquid $\mathrm{Zr}_{46.8} \mathrm{Ti}_{8.2} \mathrm{Cu}_{7.5} \mathrm{Ni}_{10} \mathrm{Be}_{27.5}$ and that it in full accord with mode-coupling theory. The dynamics in an equilibrium metallic liquid towards longer times as well as its behavior relative to the structural $\alpha$ relaxation known from nonmetallic glass-forming systems, remains an open question.

In this Letter we report on the study of structural relaxation in liquid $\mathrm{Pd}_{40} \mathrm{Ni}_{10} \mathrm{Cu}_{30} \mathrm{P}_{20}$ over a broad temperature range. This alloy is the best metallic glass former known so far. A critical cooling rate of only $0.1 \mathrm{~K} / \mathrm{s}$ is sufficient to avoid crystallization and to obtain bulk metallic glass castings of up to $72 \mathrm{~mm}$ in diameter [9]. $\mathrm{Pd}_{40} \mathrm{Ni}_{10} \mathrm{Cu}_{30} \mathrm{P}_{20}$ combines a deep eutectic composition with a liquidus temperature $T_{\text {liq }}$ at only $865 \mathrm{~K}(V 4: 1050 \mathrm{~K})$ with a high glass transition temperature $T_{g} \simeq 578 \mathrm{~K}(V 4: \simeq 626 \mathrm{~K})$. Therefore, in contrast to simple metallic liquids and most alloys, relaxational dynamics in liquid $\mathrm{Pd}_{40} \mathrm{Ni}_{10} \mathrm{Cu}_{30} \mathrm{P}_{20}$ is sufficiently slow to study structural relaxation in an equilibrium metallic liquid with quasielastic neutron scattering.

Common features of structural $\alpha$ relaxation in glassforming liquids are a universal temperature dependence, where correlation functions obey a time-temperature superposition, and a stretching over a wider time range than expected for exponential relaxation [8,10]. Experimental data in the $\alpha$ relaxation regime can usually be well described by a Kohlrausch stretched exponential function

$$
F(q, t)=f_{q} \exp \left(-t / \tau_{q}\right)^{\beta_{q}}
$$

with an exponent $\beta_{q}<1 ; \tau_{q}$ is the relaxation time and $f_{q}$ accounts for the initial decay of correlations due to phonons and a fast relaxation process.

We investigated structural relaxation in liquid $\mathrm{Pd}_{40} \mathrm{Ni}_{10} \mathrm{Cu}_{30} \mathrm{P}_{20}$ on the neutron time-of-flight spectrometer IN 6 at the Institut Laue-Langevin in Grenoble. In the setup used, an incident neutron wavelength of $\lambda=5.1 \AA^{-1}$ yielded an accessible wave-number range at a zero energy transfer of $q=0.3-1.5 \AA^{-1}$ at an energy resolution of $94 \mu \mathrm{eV}$ (FWHM). Regarding the scattering cross sections of the individual elements, $\mathrm{Pd}_{40} \mathrm{Ni}_{10} \mathrm{Cu}_{30} \mathrm{P}_{20}$ is an $88 \%$ coherent scatterer. However, with the first structure factor maximum at $q_{0} \simeq 2.9 \AA^{-1}$, our spectra are dominated by incoherent scattering, which in turn is dominated by the contributions from $\mathrm{Ni}$ with $\simeq 73 \%$ and $\mathrm{Cu}$ with $\simeq 22 \%$.

$\mathrm{Pd}_{40} \mathrm{Ni}_{10} \mathrm{Cu}_{30} \mathrm{P}_{20}$ ingots were prepared from a mixture of pure elements, and $\mathrm{NiP}$ and $\mathrm{CuP}$ alloys by induction 
melting in a silica tube. The melt was subject to a $\mathrm{B}_{2} \mathrm{O}_{3}$ flux treatment in order to improve its glass-forming ability. Differential scanning calorimetry with a heating rate of $40 \mathrm{~K} / \mathrm{min}$ resulted in a $T_{g}$ at $578 \mathrm{~K}$ and a $T_{\text {liq }}$ at $865 \mathrm{~K}$ in accordance with values given in Ref. [9]. Slices with a thickness of $1.5 \mathrm{~mm}$ were cut from the amorphous bulk sample and sealed in a $\mathrm{SiC}$ container.

Spectra of liquid $\mathrm{Pd}_{40} \mathrm{Ni}_{10} \mathrm{Cu}_{30} \mathrm{P}_{20}$ were taken at five temperatures above $T_{\text {liq }}=865 \mathrm{~K}$ ranging from $873 \mathrm{~K}$ to $1033 \mathrm{~K}$. Additional spectra were measured in the undercooled liquid at $838 \mathrm{~K}$ without evidence of crystallization. Measurements of the amorphous sample at $300 \mathrm{~K}$ gave the instrumental resolution profile. The high flux on IN 6 allowed for relatively short measuring times of $2.5 \mathrm{~h}$ at each temperature. In order to obtain the scattering law $S(q, \omega)$, raw data were normalized to a vanadium standard, corrected for self-absorption and container scattering, and interpolated to constant wave numbers $q$. Multiple scattering corrections were omitted, since the sample scatters less than $1 \%$. Symmetrization of $S(q, \omega)$ with the detailed balance factor, Fourier transformation, division by the instrumental resolution, and normalization to the $t=0$ value yielded the correlation function $\Phi(q, t)$ up to $10 \mathrm{ps}$.

Figure 1 shows the scattering law $S(q, \omega)$ at $q=$ $1.5 \AA^{-1}$ - for clarity, normalized to the value at $\omega=0$. Structural relaxation in liquid $\mathrm{Pd}_{40} \mathrm{Ni}_{10} \mathrm{Cu}_{30} \mathrm{P}_{20}$ is characterized by a quasielastic signal with a remarkable small width of some $10 \mu \mathrm{eV}$ (FWHM) at about $T_{\text {liq }}, 2-$ 3 orders of magnitude smaller than in simple metallic liquids at their melting points. The wings of the quasielastic lines extend up to several $100 \mu \mathrm{eV}$.

For a quantitative analysis we turn to the correlation function $\Phi(q, t)$ obtained by Fourier deconvolution of measured $S(q, \omega)$. Figure 2 shows $\Phi(q, t)$ in a semilogarithmic representation from $t \simeq 0.4$ up to $10 \mathrm{ps}$ and at $q=$ $1.5 \AA^{-1}$. For $t>1 \mathrm{ps}$, structural relaxation dominates the spectral line shape. We note a stretching in $\Phi(q, t)$. Solid lines in Fig. 2 are fits with a Kohlrausch stretched

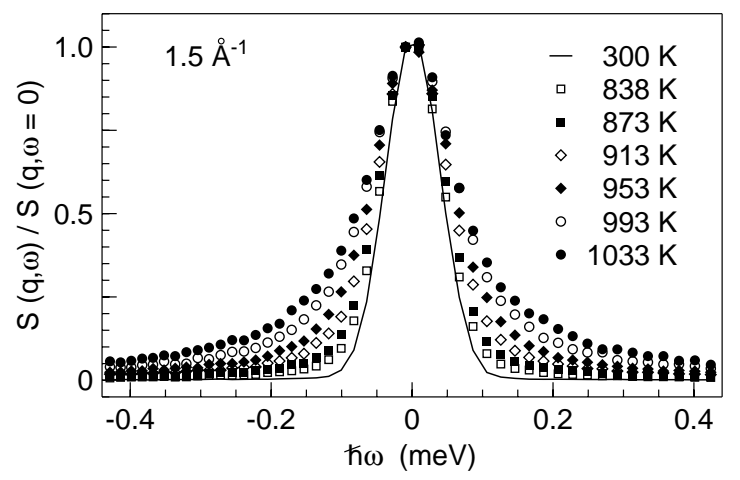

FIG. 1. Scattering law $S(q, \omega)$ of $\mathrm{Pd}_{40} \mathrm{Ni}_{10} \mathrm{Cu}_{30} \mathrm{P}_{20}$ measured with quasielastic neutron scattering on the time-of-flight spectrometer IN 6. The measurement in the glass at $300 \mathrm{~K}$ yields the instrumental resolution function. Structural relaxation causes a broadening of the elastic line on a $10 \mu \mathrm{eV}$ scale - some 3 orders of magnitude smaller than in simple metallic liquids at their melting points. exponential function [Eq. (1)] in the time range from 1 to 10 ps. Towards shorter times, $\Phi(q, t)$ reaches a plateau; data are still well described by the stretched exponential function. This demonstrates that the microscopic dynamics are well separated from the quasielastic signal [11].

The correlation function $\Phi(q, t)$ can be fit using Eq. (1) with the stretching exponent $\beta_{q}$ as a free parameter for wave numbers ranging from 0.9 to $1.5 \AA^{-1}$. Although results for $\beta_{q}$ scatter between 0.70 and 0.82 , no systematic $q$ or temperature dependence of $\beta_{q}$ is found. Figure 3(a) displays master curves constructed with values $f_{q}$ and $\tau_{q}$ obtained with a mean $\beta=0.76$. Time-temperature superposition holds over the entire temperature range, even far above the melting point. This is in agreement with results from quasielastic neutron scattering on the glassforming liquids glycerol [12] and orthoterphenyl [13]. Stretching of correlation functions is generally found to be more pronounced in fragile glass-forming liquids, characterized by a sharp temperature dependence of viscosity, e.g., the van der Waals liquid orthoterphenyl with a $\beta \simeq 0.5$ [13]. In an intermediate system such as glycerol, a $\beta \simeq 0.6$ has been reported [12]. Hence, a stretching exponent of $\beta \simeq 0.76$ suggests that $\mathrm{Pd}_{40} \mathrm{Ni}_{10} \mathrm{Cu}_{30} \mathrm{P}_{20}$ is a fairly strong glass former.

In a second fitting iteration we used an average over all values $\overline{\beta_{q}(T)}=\beta=0.76 \pm 0.02$, which is then kept fixed. The resulting mean relaxation times

$$
\left\langle\tau_{q}\right\rangle=\int_{0}^{\infty} d t F(q, t) / f_{q}=\tau_{q} \beta^{-1} \Gamma\left(\beta^{-1}\right) .
$$

are proportional to $1 / q^{2}$ within $5 \%$, as one would expect in the hydrodynamic limit for $q \ll q_{0}$ [14]. This permits the determination of an average diffusivity $D=$ $1 / q^{2}\left\langle\tau_{q}\right\rangle$ [Fig. 3(b)]. Values range from $0.44 \pm 0.02 \times$ $10^{-10} \mathrm{~m}^{2} \mathrm{~s}^{-1}$ at $838 \mathrm{~K}$ to $4.9 \pm 0.1 \times 10^{-10} \mathrm{~m}^{2} \mathrm{~s}^{-1}$ at $1033 \mathrm{~K}$. Even $168 \mathrm{~K}$ above the liquidus temperature, long-range atomic motion in liquid $\mathrm{Pd}_{40} \mathrm{Ni}_{10} \mathrm{Cu}_{30} \mathrm{P}_{20}$ is approximately 2 orders of magnitude slower than in simple metallic liquids.

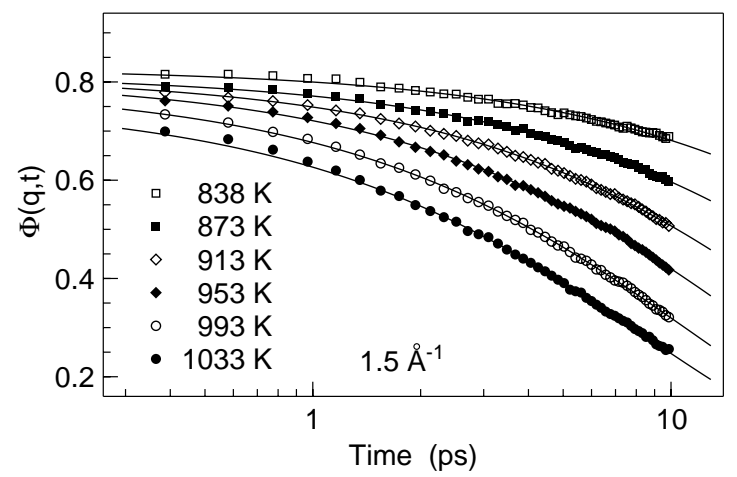

FIG. 2. Normalized time correlation function $\Phi(q, t)$ of liquid $\mathrm{Pd}_{40} \mathrm{Ni}_{10} \mathrm{Cu}_{30} \mathrm{P}_{20}$ obtained by Fourier deconvolution of measured $S(q, \omega)$. Solid lines are fits with a Kohlrausch stretched exponential function resulting in a $q$ and temperatureindependent stretching exponent $\beta=0.76 \pm 0.02$. Even far above the liquidus temperature $T_{\text {liq }}=865 \mathrm{~K}$, structural relaxation remains nonexponential. 


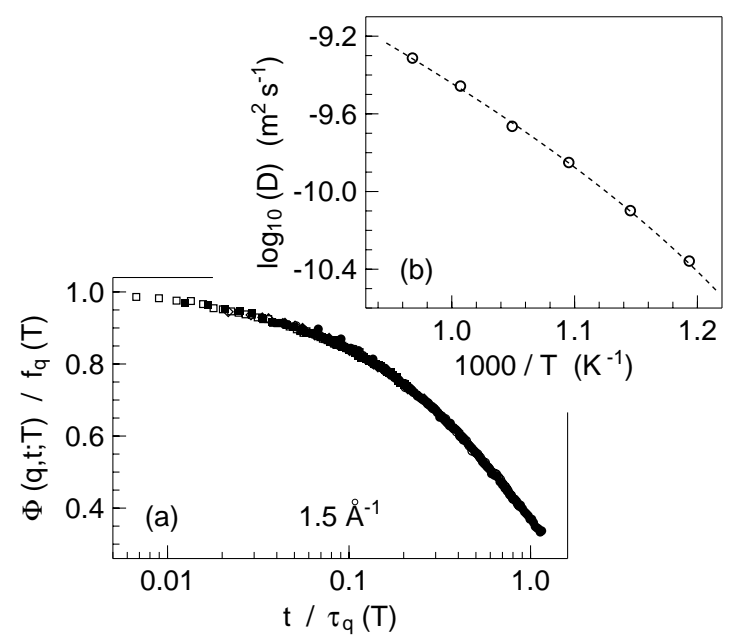

FIG. 3. Structural relaxation in liquid $\mathrm{Pd}_{40} \mathrm{Ni}_{10} \mathrm{Cu}_{30} \mathrm{P}_{20}$ : (a) Rescaling of the correlation function $\Phi(q, t)$ at $q=1.5 \AA^{-1}$ (Fig. 2) using results from fits with a stretched exponential function and a mean $\beta=0.76-$ a time-temperature superposition holds. (b) Diffusivity $D=1 / q^{2}\left\langle\tau_{q}\right\rangle$ calculated from the mean relaxation times. Its temperature dependence is in accord with mode-coupling theory (dashed line) indicating a crossover temperature at $T_{c} \simeq 695 \pm 30 \mathrm{~K}$.

Mode-coupling theory is primarily concerned with short-time dynamics around the fast $\beta$ relaxation regime. However, it also provides predictions for the temperature dependence of the $\alpha$ relaxation time. Within mode-coupling theory, $T_{c}$ is the temperature at which the transport mechanism changes from glasslike hopping to liquidlike motion, i.e., viscous flow. In the idealized version of the theory, which does not account for hopping processes, $\tau$ is directly proportional to $\left[\left(T-T_{c}\right) / T_{c}\right]^{-\gamma}$ for temperatures above $T_{c}$ [8]. Although MCT does not make predictions of the temperature range in which this relation holds, it allows a rough estimate of the crossover temperature $T_{c}$. A best fit to $D(T)$ yields a $T_{c}=695 \pm 30 \mathrm{~K}$ and a $\gamma=2.8 \pm 0.2$. The exponent compares to the value found in liquid $\mathrm{Zr}_{46.8} \mathrm{Ti}_{8.2} \mathrm{Cu}_{7.5} \mathrm{Ni}_{10} \mathrm{Be}_{27.5}(\gamma=2.7)$ derived from the line shape analysis of $\Phi(q, t)$ in the fast $\beta$ relaxation regime [6]. The MCT power law also holds for self-diffusion coefficients obtained by molecular dynamics simulations in binary $\mathrm{ZrNi}$ and $\mathrm{ZrCu}$ alloys $[15,16]$.

In liquid $\mathrm{Zr}_{46.8} \mathrm{Ti}_{8.2} \mathrm{Cu}_{7.5} \mathrm{Ni}_{10} \mathrm{Be}_{27.5}$, we extrapolated a $T_{c}$ to $\simeq 1.5 T_{g}$. Unfortunately, $T_{c}$ lies in the inaccessible temperature range between crystallization and melting. In $\mathrm{Pd}_{40} \mathrm{Ni}_{10} \mathrm{Cu}_{30} \mathrm{P}_{20}, T_{c}\left(\leq 1.3 T_{g}\right)$ is much closer to the glass transition, and the thermal stability against crystallization is even greater. For this alloy system a measurement of critical parameters around the crossover from an ergodic to a nonergodic metallic liquid may thus be possible.

In conclusion, long-range atomic motion in liquid $\mathrm{Pd}_{40} \mathrm{Ni}_{10} \mathrm{Cu}_{30} \mathrm{P}_{20}$ occurs on a $10^{-10} \mathrm{~m}^{2} \mathrm{~s}^{-1}$ scale, some 3 orders of magnitude slower than in simple metallic liquids. The quasielastic signal obeys a universal timetemperature superposition for temperatures ranging from
$T_{\text {liq }}-27 \mathrm{~K}$ to $T_{\text {liq }}+168 \mathrm{~K}$. Liquid $\mathrm{Pd}_{40} \mathrm{Ni}_{10} \mathrm{Cu}_{30} \mathrm{P}_{20}$ exhibits a structural $(\alpha)$ relaxation like other nonmetallic viscous liquids. The structural relaxation is nonexponential-it shows a stretching in time with a stretching exponent $\beta=0.76 \pm 0.02$, indicating that metallic bulk-glass forming $\mathrm{Pd}_{40} \mathrm{Ni}_{10} \mathrm{Cu}_{30} \mathrm{P}_{20}$ is a fairly strong glass-forming liquid.

We thank W.L. Johnson and W. Petry for their continuous support, J.F. Löffler for his help with the sample preparation, and W. A. Kamitakahara for a critical reading of the manuscript.

*Permanent address: Physik Department E 13, Technische Universität München, 85747 Garching, Germany.

Electronic address: ameyer@ph.tum.de

${ }^{\dagger}$ Present address: Department of Mechanical Engineering, 204 Rogers Hall, Oregon State University, Corvallis, Oregon 97331.

[1] T. Zhang, A. Inoue, and T. Masumoto, Mater. Trans. JIM 32, 1005 (1991).

[2] A. Peker and W. L. Johnson, Appl. Phys. Lett. 63, 2342 (1993).

[3] R. Busch, E. Bakke, and W. L. Johnson, Acta Mater. 46, 4625 (1998).

[4] A. Masuhr, T. A. Waniuk, R. Busch, and W. L. Johnson, Phys. Rev. Lett. 82, 2290 (1999).

[5] U. Balucani and M. Zoppi, Dynamics of the Liquid State (Clarendon, Oxford, 1994).

[6] A. Meyer, J. Wuttke, W. Petry, O. G. Randl, and H. Schober, Phys. Rev. Lett. 80, 4454 (1998); A. Meyer, J. Wuttke, and W. Petry, J. Non-Cryst. Solids 250-252, 116 (1999).

[7] For an overview of dynamics in glass-forming liquids and a comparison of experiments with theoretical predictions, see H.Z. Cummins, G. Li, Y.H. Hwang, G. Q. Shen, W. M. Du, J. Hernandez, and N. J. Tao, Z. Phys. B 103, 501 (1997).

[8] W. Götze and L. Sjögren, Rep. Prog. Phys. 55, 241 (1992); for an overview of recent tests of the theory, see W. Götze, J. Phys. Condens. Matter 11, A1 (1999).

[9] A. Inoue, N. Nishiyama, and H. Kimura, Mater. Trans. JIM 38, 179 (1997).

[10] J. Wong and C.A. Angell, Glass Structure by Spectroscopy (Marcel Dekker, New York, 1976).

[11] This paper focused on structural relaxation and, therefore, was not optimized for the investigation of a fast $\beta$ process. However, the fast $\beta$ relaxation in $\mathrm{Pd}_{40} \mathrm{Ni}_{10} \mathrm{Cu}_{30} \mathrm{P}_{20}$ appears to be appreciably weaker than in $\mathrm{Zr}_{46.8} \mathrm{Ti}_{8.2} \mathrm{Cu}_{7.5} \mathrm{Ni}_{10} \mathrm{Be}_{27.5}$. Further experiments to clarify this interesting difference are in preparation.

[12] J. Wuttke, I. Chang, O. G. Randl, F. Fujara, and W. Petry, Phys. Rev. E 54, 5364 (1996).

[13] A. Tölle, J. Wuttke, H. Schober, O.G. Randl, and F. Fujara, Eur. Phys. J. B 5, 231 (1998).

[14] J.P. Boon and S. Yip, Molecular Hydrodynamics (McGraw-Hill, New York, 1980).

[15] H. Teichler, Defect Diffus. Forum 143-147, 717 (1997).

[16] C. Gaukel and H. R. Schober, Solid State Commun. 107, 1 (1998). 